However, little is known about variation in swimming performance among different morphs, in labriform swimmers. The first aim of our study was to examine the relationships between anaerobic swimming performance and individual morphometric measurements in the shiner perch. Shiner perch (Cymatogaster aggregata), is a conspicuous and abundant surf perch of the Pacific Northwest that lives in complex habitat. Fast start responses of 40 fish were first recorded. Latency (time from stimulus to visible response movement) and coordinate data were collected for the center of mass and snout positions. From these coordinates, smoothed velocity, acceleration profiles, turning radii and turning angles were calculated. Then 14 morphologic characteristics were measured on each fish (body shape, tail shape, white muscle). The second objective of this study was to determine the temporal stability of fast start performance. Thus, we repeated 4 successive tests on each 40 fish.

doi:10.1016/j.cbpa.2007.01.225

\section{A6.52 \\ Regional variation in the microhardness and mineral content of porcine long bones}

H. Bonney, A. Goodman, B. Colston, (University of Lincoln, United Kingdom)

Pig carcasses are frequently used as models for the human cadaver in the study of trauma and wound patterns. Limited availability and ethical concerns over the use of human tissue has meant that porcine bone is often used as a substitute for human bone in such studies. However, there is a lack of comprehensive mechanical data to make informed decisions on the choice of model to account for variations in the age, sex and body mass.

In this study, variation in the properties of cortical bone along the length of the porcine humerus and ulna, from five female pigs aged over 36 months, was investigated by using Vickers microhardness tests. Transverse sections were taken from the proximal, mid and distal regions of the diaphyses, and tests were carried out in the anterior, medial, posterior, and lateral quadrants. Microhardness was also measured across the cortex from the periosteal to endosteal surfaces. Regional variation in mineral content was explored by analysing samples, taken immediately adjacent to the hardness tests, using Atomic Absorption Spectroscopy.

Mechanical data are discussed in relation to mineral content, morphology and loading of the limbs, within a forensic context.

doi:10.1016/j.cbpa.2007.01.226

\section{A6.53}

How do tree frogs adhere well to smooth surfaces and yet detach easily when necessary?

\section{J. Platter, J. Pearman, J. Barnes, (Glasgow University, Scotland)}

Tree frogs adhere well to smooth surfaces even when they are tilted well beyond the vertical. Some small species can even adhere when completely upside-down. Yet, when walking or jumping, tree frogs detach their toe pads easily without significant pull-off forces. Detachment has previously been shown to occur by peeling (Hanna and Barnes, 1991), but how is peeling controlled? Why doesn't a tree frog on an overhanging surface simply peel off rather than adhere? To investigate this, we have recorded adhesive and shear (frictional) forces simultaneously from single toe pads in the hylid tree frog, Litoria caerulea. By varying the angle of pull, we were able to examine the effect of angle on both force components and test whether there is a particular range of angles at which peeling takes place. From these data, it appears that there is no rapid transition from adhesion to peeling. However, adhesive forces decline gradually with increasing angles of pull, particularly for angles beyond $90^{\circ}$, and are negligible above $150^{\circ}$. Thus frogs maintain a grip by keeping the angle of their toes with respect to the substrate at a low value, and detach by increasing this angle to beyond $90^{\circ}$. These data are supported by behavioural observations on tree frogs adhering to smooth surfaces that are tilted from $0^{\circ}$ to $180^{\circ}$. At increasing angles, legs are spread out sideways so that angles between feet and substrate are kept as low as possible.

Hanna, G. and Barnes, W.J.P., 1991. J. Exp. Biol., 155, $103-125$.

doi:10.1016/j.cbpa.2007.01.227

\section{A6.54 \\ Design of a laminar flow tank for studying the development of zebra fish swimming}

G. de Rue, D. Lentink, (Wageningen University, The Netherlands)

The hydrodynamics of undulatory swimming are still poorly understood. Different gaits and corresponding wake structures may have evolved for energetic efficiency, but could also result from other developmental, evolutionary or hydrodynamic constraints. To research this in detail we set out to develop a swim tunnel with as laminar a flow field as possible for PIV and kinematics research. The setup accommodates Zebra fish (Danio rerio) of different ages at Reynolds numbers ranging from 30 to 30000 . For flow analysis background vorticity and turbulence must be minimised to adequately visualise and study the wake structures.

Three basic designs were analysed: A classical pump driven pipe flow tunnel, a caterpillar driven concentric tank, and a spinning annular flow tank; a fish wheel.

To gain insight into their differences, we analysed the three designs by means of 2D CFD simulations. The results show that the classic tunnel design suffers from excessive boundary layers at low speeds and will become turbulent at higher Reynolds numbers. The caterpillar tank produces a large amount of 